\title{
Engineered Structured Sorbents for the Adsorption of Carbon Dioxide and Water Vapor from Manned Spacecraft Atmospheres: Applications and Modeling 2007/2008
}

\author{
James C. Knox \\ NASA, Marshall Space Flight Center \\ David F. Howard \\ All Points Logistics, Inc \\ Jay L. Perry \\ NASA, Marshall Space Flight Center
}

\begin{abstract}
In NASA's Vision for Space Exploration, humans will once again travel beyond the confines of earth's gravity, this time to remain there for extended periods. These forays will place unprecedented demands on launch systems. They must not only blast out of earth's gravity well as during the Apollo moon missions, but also launch the supplies needed to sustain a larger crew over much longer periods. Thus all spacecraft systems, including those for the separation of metabolic carbon dioxide and water from a crewed vehicle, must be minimized with respect to mass, power, and volume. Emphasis is also placed on system robustness both to minimize replacement parts and ensure crew safety when a quick return to earth is not possible.
\end{abstract}

This paper describes efforts to improve on typical packed beds of sorbent pellets by making use of structured sorbents and alternate bed configurations to improve system efficiency and reliability. The development efforts described offer a complimentary approach combining testing of subscale systems and multiphysics computer simulations to characterize the regenerative heating substrates and evaluation of engineered structured sorbent geometries. Mass transfer, heat transfer, and fluid dynamics are included in the transient simulations.

\section{INTRODUCTION}

Beginning in the 2020 timeframe, NASA plans to launch a new era of lunar exploration that includes building a lunar outpost that will be staffed continuously by four astronauts on six-month shifts. Further in the future, the U.S. space exploration policy calls for a manned mission to Mars; a two to three-year roundtrip.
An engineering requisite for all space travel is to minimize power, weight, and volume because all three translate to mass for any launch system. Compared to near-earth missions such as the International Space Station, manned lunar outposts and long-duration space travel present additional constraints that stress system engineering. Chief among these constraints is that every system must be robust enough to operate for long periods of time without compromising crew safety, without re-supply, and without launch-taxing extra mass such as spare parts or a glut of backup equipment. Life support systems are no exception.

The current state of the art $\mathrm{CO}_{2}$ removal system onboard the International Space Station is not only large in mass, but has also proven to be problematic with the majority of issues related to breakdown of sorbent pellets, generating dust that clogs and contaminates the system. To improve upon existing sorbent based systems that use beds packed with pellets of sorbent material, an effort to develop and assess structured sorbents to include single piece extruded lattices, Robocast formed activated alumina, silica gel beads packed in aluminum foam, and sorbent coated metals are under evaluation. These systems offer the benefit of higher structural stability and potential gains in operational efficiencies.

\section{CURRENT LIFE SUPPORT TECHNOLOGY}

The average person exhales about $1 \mathrm{~kg}$ of $\mathrm{CO}_{2}$ and sheds about $2.33 \mathrm{~kg}$ of water as part of their normal daily metabolic process, with higher emission rates during strenuous physical activity. It is paramount that these parameters are controlled because at high levels $\mathrm{CO}_{2}$ can be toxic and condensation forming on electronics can damage systems vital to the mission. The International Space Station has been continuously inhabited for more than 6 years by at least two 
crewmembers, and sometimes as many as 10 when the Space Shuttle docks. The current life-support technology is a four-bed molecular sieve (4BMS) shown in Figure 1. This system dries the process air prior to $\mathrm{CO}_{2}$ removal and returns the captured water vapor back to the cabin air. This system currently vents $\mathrm{CO}_{2}$, although with modifications it could store $\mathrm{CO}_{2}$ in an accumulator for subsequent water production. Highly effective, the 4BMS removes 100 percent of the metabolic $\mathrm{CO}_{2}$ for up to six crew as well as some trace gases emitted by people and equipment [1].

The 4BMS system, however, has a number of drawbacks, making it unsuitable for extended stays beyond low earth orbit. One of these shortcomings is due to the poor thermal conductivity of molecular sieves. As a result, indirect heating to assist desorption is inherently inefficient, requiring large amounts of power. The 4BMS also uses packed beds of pelletized sorbent materials, which tend to generate dust.

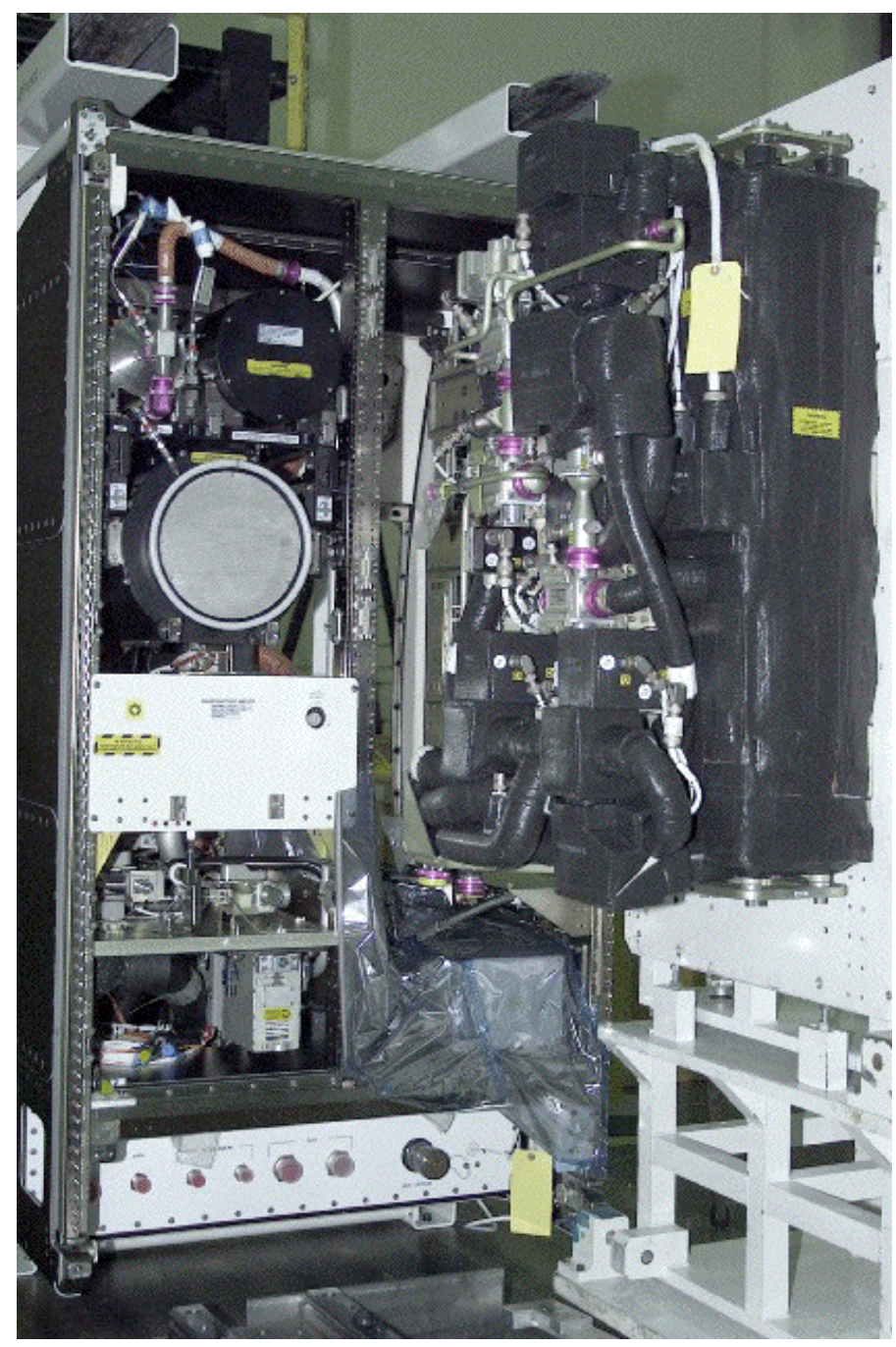

Figure 1: The four-bed molecular sieve (4BMS) carbon dioxide removal assembly used in the International Space Station

Limitations such as these have led to exploring new designs for long-duration space travel. Solid sorbents such as zeolites coated on expanded metal or metal lattices can be directly heated by sending an electrical current through the metallic substrate. This approach also provides a dust-free adsorption process for the removal of metabolic $\mathrm{CO}_{2}$ and water.

\section{STRUCTURED SORBENT TECHNOLOGIES}

Engineered Structured Sorbent (ESS) technologies currently under evaluation include single piece sorbent lattice structures, sorbent coatings over metallic substrates, and silica gel media packed within an aluminum foam structure. Each form of structured sorbent offers its own advantages and disadvantages that will determine it's feasibility for use in any specific application. To make this determination each technology is slated for evaluation by either testing using subscale technology samples, computer simulations, or both. Each technology, their potential advantages/disadvantages, their evaluations to date, and most promising applications are discussed below.

\section{SORBENT COATED METALS}

Use of a metallic substrate coated with a sorbent offers the advantages of greater structural stability compared to packed beds, and also had the advantage of the material being in direct contact to either a high thermally conductive material, or a material used for a resistive heating element. The contact with such a material is important in that it can aid the sorption processes by quickly transporting heat into or away from the material to either drive or enhance the process.

\section{$\underline{\text { Microlith }^{\mathrm{TM}}}$}

One of the coated technologies currently under evaluation is the sorbent version Microlith ${ }^{\mathrm{TM}}$ developed by Precision Combustion Incorporated (PCl) [2,3] The sorbent version is an extension of successful work on Microlith $^{\mathrm{TM}}$ based oxidation catalysts [4]. The sorbent version of the technology consists of sorbent material coated onto an expanded metal screen. In this application, the screen acts as a base structure as well as a heating element. The major issue to overcome with this technology is the different coefficients of thermal expansion between the expanded metal and the sorbent coating. To remedy this $\mathrm{PCl}$ developed a proprietary coating technique that has proven to retain the sorbent through 30 cycles consisting of over 530 hours. Figures $2 \mathrm{a}, 2 \mathrm{~b}$, and $2 \mathrm{c}$ demonstrate the Microlith ${ }^{\mathrm{TM}}$ construction. 


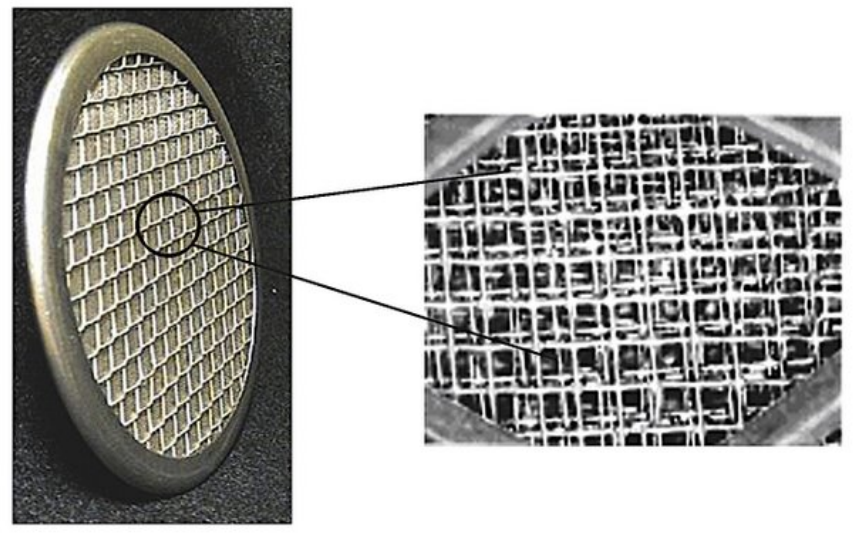

Figure 2A: Microlith $^{\mathrm{TM}}$ oxidation catalyst version of expanded metal screen with close up view

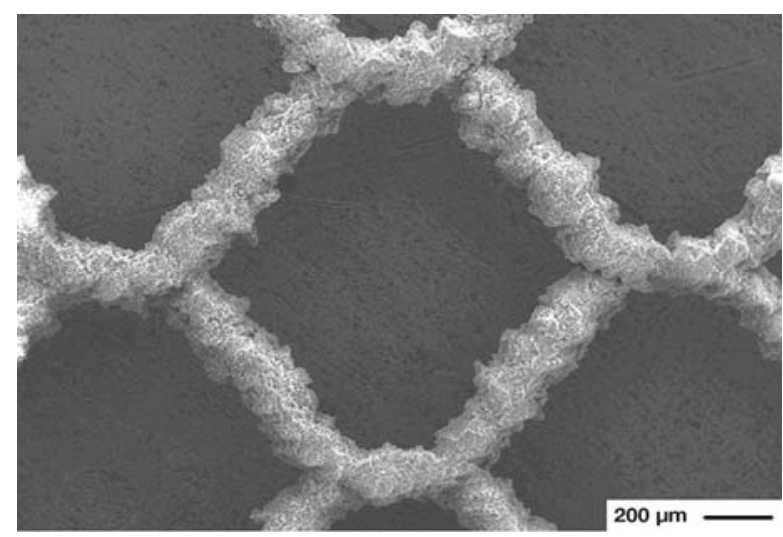

Figure 2B: Micrograph of sorbent-coated Microlith $^{\mathrm{TM}}$

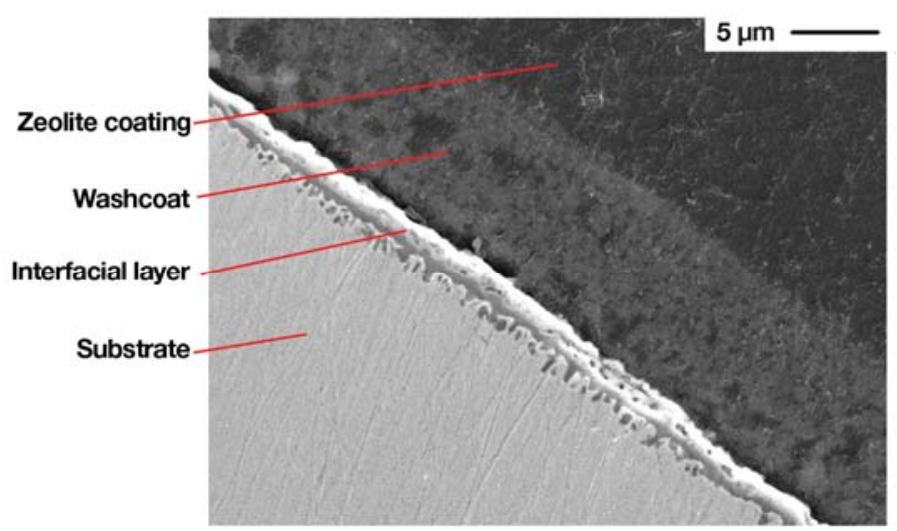

Figure 2C: Sorbent Coating Layering

The primary configuration under evaluation consists of a length of coated screen and a thin layer of fiberglass insulation rolled into a cylindrical shape to effectively produce a radial stack of screen layers, each separated by a thin layer of insulation. Air is processed by flowing into the center of the cylinder and dispersing radially through the material. This configuration is demonstrated in Figure 3.

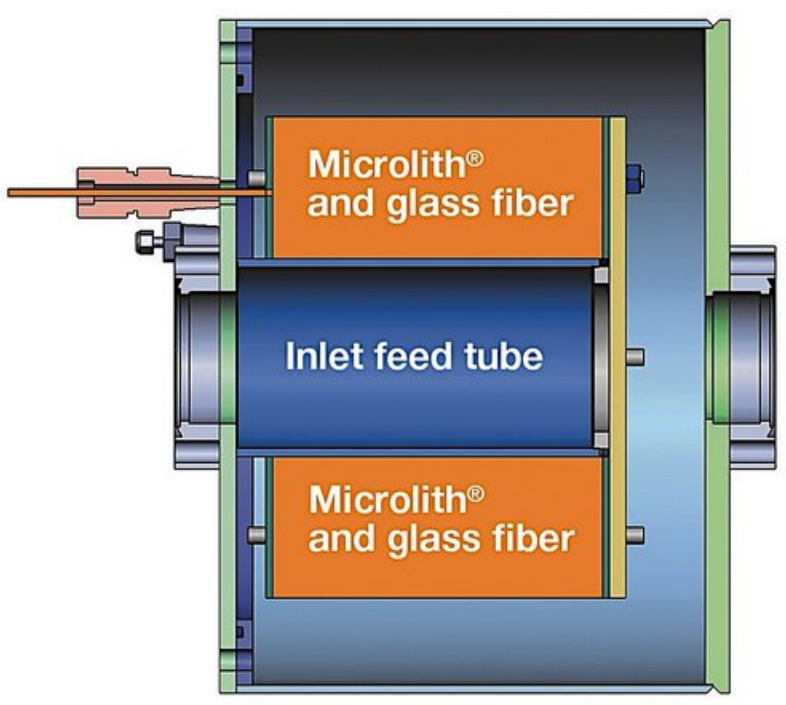

Figure 3 - Radial Flow Microlith ${ }^{\mathrm{TM}}$ Packaging.

Sorbent-coated metal technologies present a number of tradeoffs in terms of working capacity, mass, and volume. Subscale testing and Multiphysics simulations play a key role in the design and analysis process performance as we evaluate the technology.

Evaluation of this technology to date is limited to the flow dispersion through the material using a computer simulation. As shown in Figure 4, the simulation predicts a uniform gas flow through the material and the potential to significantly reduce canister volume surrounding the sorbent.

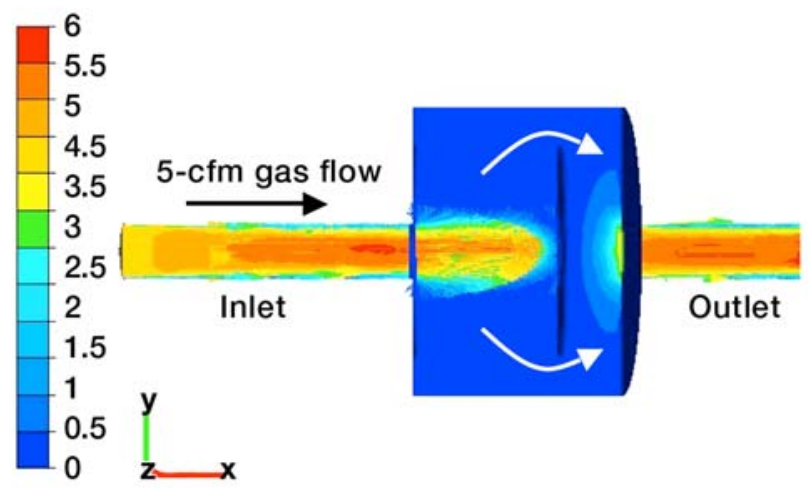

Figure 4 - Simulation showing the uniform flow of gases through the canister and the potential to reduce the flow volume

$\mathrm{PCl}$ in a collaborative manufacturing effort with NASA's Marshall Space Flight Center (MSFC), is producing three $1 / 3$ scale test articles. Each test article is specifically designed for removal of either humidity, $\mathrm{CO}_{2}$, or Trace Contaminants. The module housings were designed and fabricated by NASA MSFC and delivered to $\mathrm{PCl}$ for installation of the sorbent Microlith ${ }^{\mathrm{TM}}$ and final assembly. NASA MSFC will evaluate the modules using a test apparatus capable cycling the modules through subsequent adsorbing and desorbing cycles and 
supplying air conditioned with controlled amounts of each constituent of interest. Testing of the $\mathrm{CO}_{2}$ removal article is slated to begin in August 2008.

\section{Coated Aluminum Foam}

Another technology planned for evaluation is sorbent coating on aluminum foam. The high thermal conductivity of aluminum lends this technology to thermally linking of adjacent adsorbing and desorbing chambers, promoting an approach toward isothermal operations. Because higher temperatures result in lower sorbate loading capacities, transferring the heat generated during adsorption to an adjacent desorbing chamber will stabilize the temperatures of both processes and increase the overall working capacity of the sorbent. $\mathrm{PCl}$ has demonstrated the ability to coat aluminum foam with sorbent. A subscale 4-chamber aluminum test article configured for thermally linking has been prepared for evaluation by NASA MSFC. An example of coated aluminum foam is shown in figure 5 .

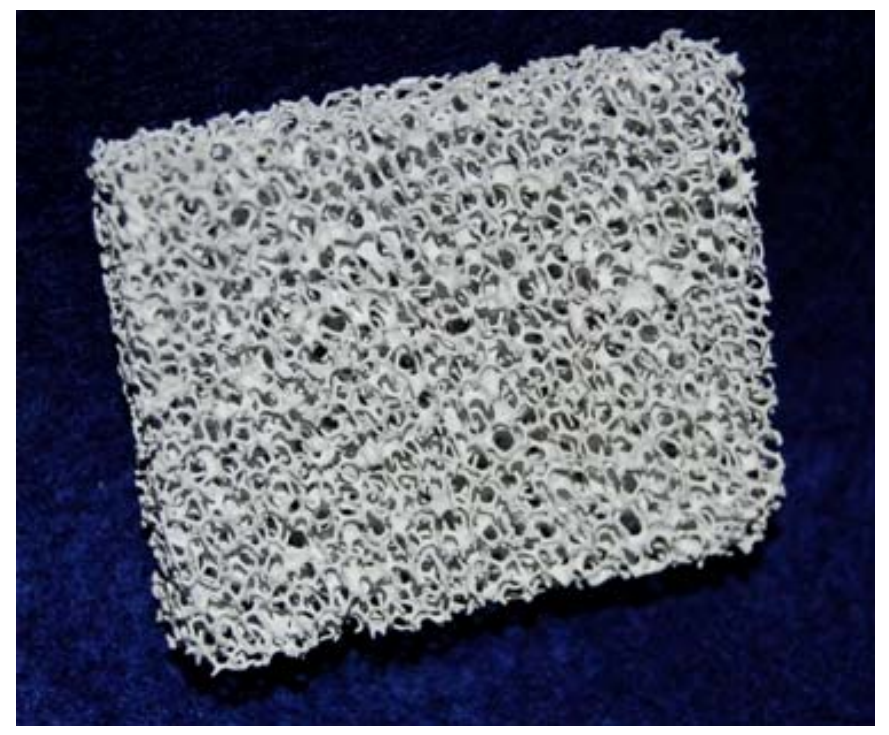

Figure 5: Sorbent Coated Aluminum Foam

The aluminum foam thermally linked approach has successfully demonstrated effective heat transfer from adjacent chambers while packed with silica gel material, and is discussed in detail in the following section.

\section{ALUMINUM FOAM PACKED WITH SILICA GEL}

A substantial improvement over standard singlechamber beds has been realized by packing silica gel sorbent beads into multi-chamber beds fitted with aluminum foam. Currently, this is the only technology being evaluated that still uses packed sorbent media opposed to single piece structures. This is acceptable because silica gel is not bound with a clay binder like the zeolite sorbent granules and does not exhibit dust producing characteristics of the zeolite materials. An example of a thermally linked chamber is shown in Figure 6.

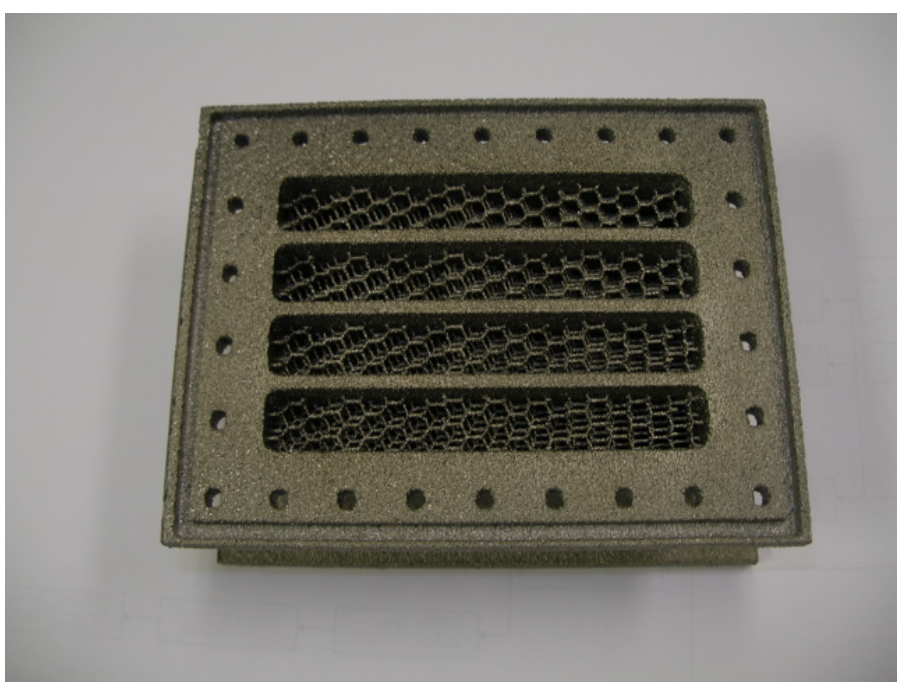

Figure 6: Four Chamber Test Article with Thermal Linking Lattice

The chamber shown in figure 6 was fabricated using an Electron Beam Melting rapid prototyping technique [5]. The lattice within each chamber is integral to the manufacturing of this article. For the Silica Gel packed in aluminum foam article, a 4-chamber bed of similar dimensions was machined from aluminum, with 10 pores per inch (ppi) aluminum foam brazed into each chamber. Silica Gel beads of $0.5 \mathrm{~mm}-0.7 \mathrm{~mm}$ was packed into the foam, and manifolds made by stereo lithography were mounted to each end to direct process air and vacuum desorption flow paths. The chamber manifold was such that adjacent chambers alternated from adsorbing to desorbing. A thermally linked chamber installed into the test stand is shown in Figure 7.

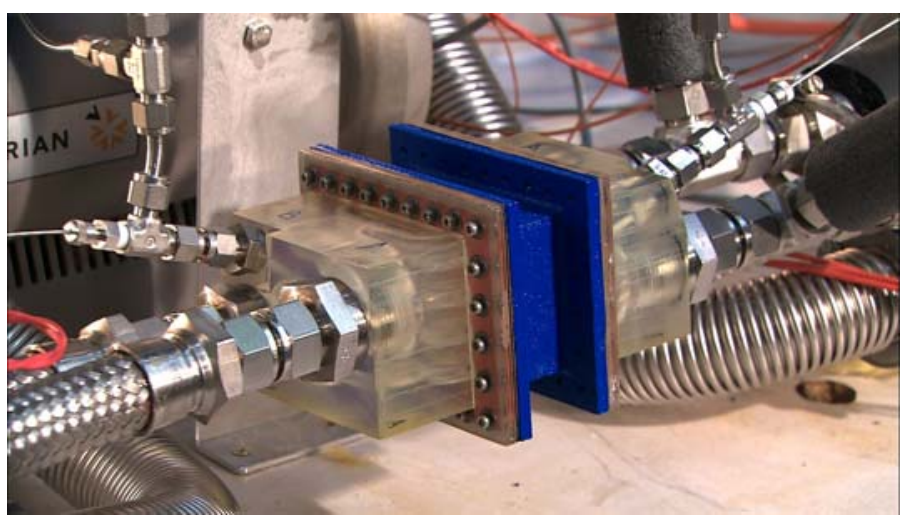

Figure 7: Thermally Linked Test Article

For comparison to thermally isolated conditions, a 4chamber test article was build out of low conductivity plastic and packed with the same size silica gel particles into chambers with no thermal carrier. Results showed that thermally linking the beds dampened temperature difference from adsorption to desorption from $15.5 \mathrm{~K}$ to $1.2 \mathrm{~K}$ during high loading cases (Figure 7). 


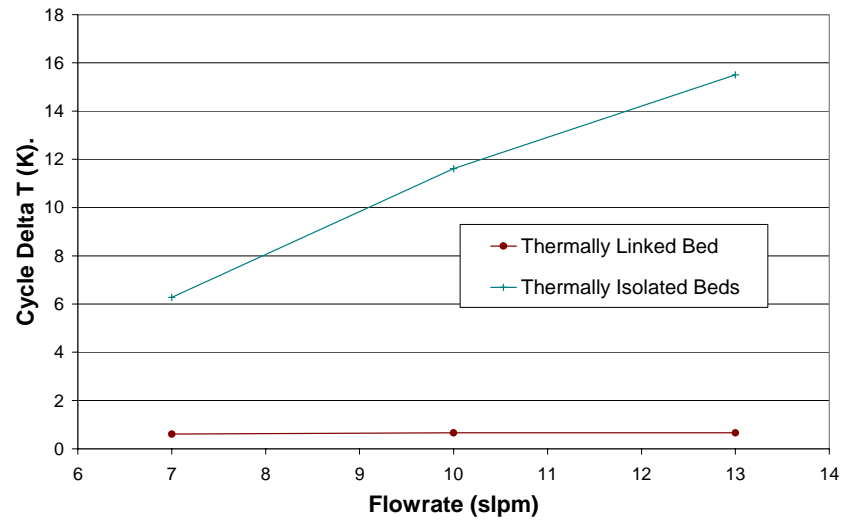

Figure 8: Temperature swings of thermally linked versus thermally isolated beds

The resulting effect substantially increased the silica gel's working capacity, enabling the cycle time to be increased from 15 to 45 minutes while still achieving at least a $90 \%$ water removal efficiency. This technology is a good candidate for a situation where a bulk water removal system is needed without water reclamation. Because it is vacuum desorbed, power consumption is extremely low. Potential uses could be in place of a condensing heat exchanger for moisture removal, or possibly for Extra Vehicular Activities (EVA's). For cases where water reclamation is required, this technology could be adapted to allow near isothermal adsorption by supplying a single chamber with convective cooling and performing thermal regeneration.

\section{MONOLITHIC SORBENT STRUCTURES}

Another technology of interest is the elimination of sorbent attrition by using a semi-open monolithic structure tailored for maximum mass transfer. The materials used in the Robocasting technique, developed by Sandia National Laboratories [6], are being expanded to include activated alumina and zeolite precursor materials. An alpha-alumina extrusion is shown in Figure 9. The lattice spacing, diameter, and offset can be adjusted to maximize surface area and volume, and minimize pressure drop.

Other monolith technologies available include extruded zeolites contained in clay binder, very similar to the composition that makes up the commercially available granules. Detailed evaluations of these sorbents are projected for 2009 .

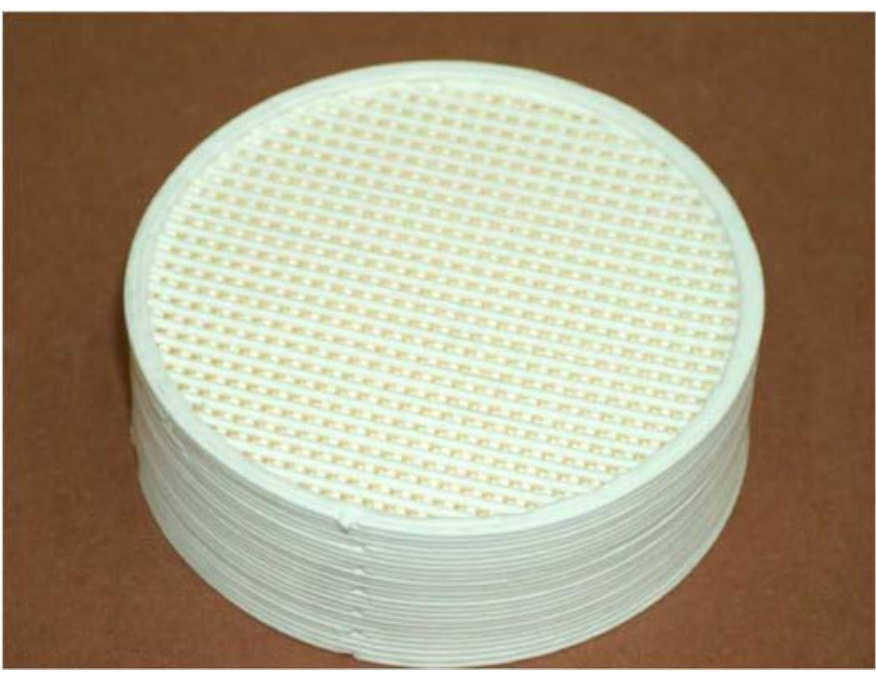

Figure 9: Activated alumina formed by Robocast ${ }^{6}$

\section{COMPUTER MODELING}

To accelerate the understanding of how the many types of structured sorbents will perform, it is desirable to use computer simulations. COMSOL Multiphysics software is currently being used to develop this capability [7]. Strides toward modeling the complex thermodynamic regenerative cycling processes have been made by first simulating breakthrough curves that correlate with previously attained test data. Isothermal adsorption testing conducted with a custom-built plate-finned heat exchanger packed with sorbent ${ }^{1}$ has been modeled (see Figure 10).

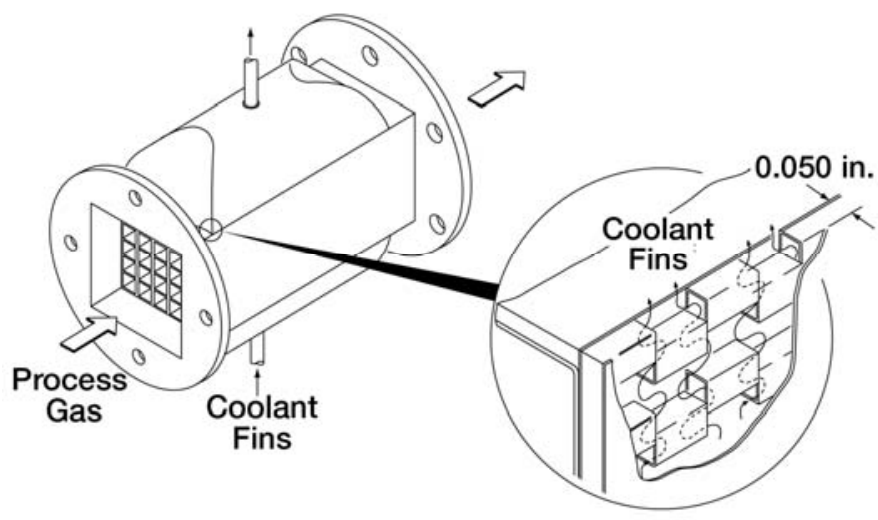

Figure 10: The packed bed heat exchanger apparatus that was used for isothermal testing

Testing began with a completely clean sorbent bed and the introduction of $\mathrm{CO}_{2}$-laden nitrogen. Initially, no $\mathrm{CO}_{2}$ exits the bed, but then the $\mathrm{CO}_{2}$ outlet history emerges in the classic S-curve shape of a breakthrough curve.

Due to the relatively constant temperature within the canister, the heat of adsorption could be neglected, allowing the mass transfer to be studied in isolation. To 
derive the Linear Driving Force (LDF) coefficient denoted as $k_{e f}$, in the solid phase, the parameter was varied until modeling predictions matched the actual test data (Figure 11). The LDF coefficient in the solid phase is the difference between the actual amount absorbed and the amount that would be absorbed at equilibrium. This value is analogous to temperature differences that provide the driving force for heat transfer.

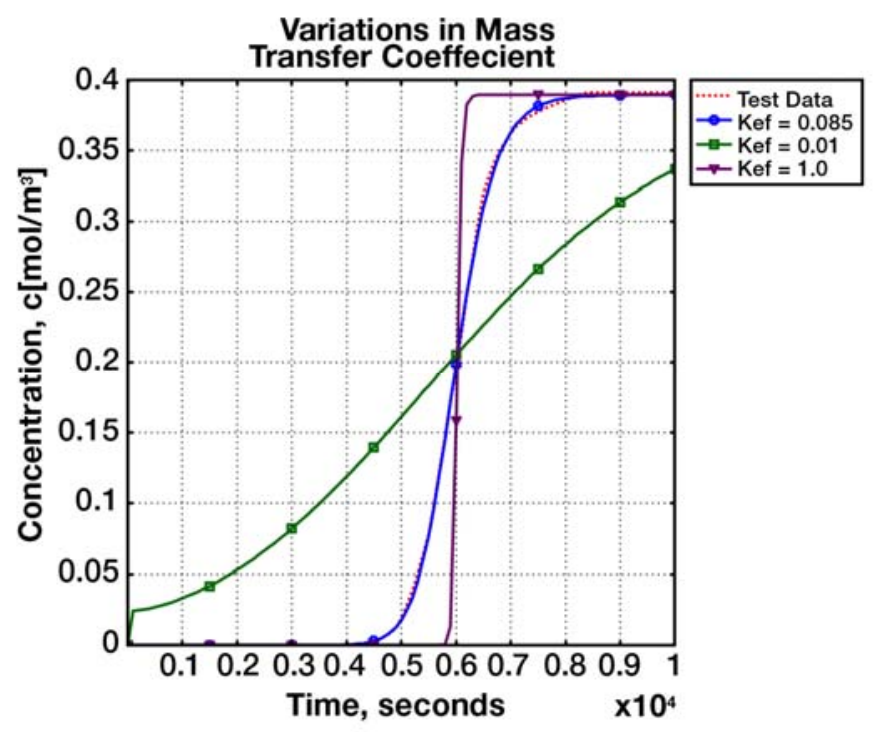

Figure 11: Actual test data shows that adjusting the LDF coefficient matched the actual test data.

The next test simulated was water adsorption on silica gel with the same apparatus (see Figure 12). After adjusting the LDF coefficient for this process, data from the COMSOL Multiphysics simulation (blue lines) once again provided a reasonable match with the actual inlet data (red dotted line).

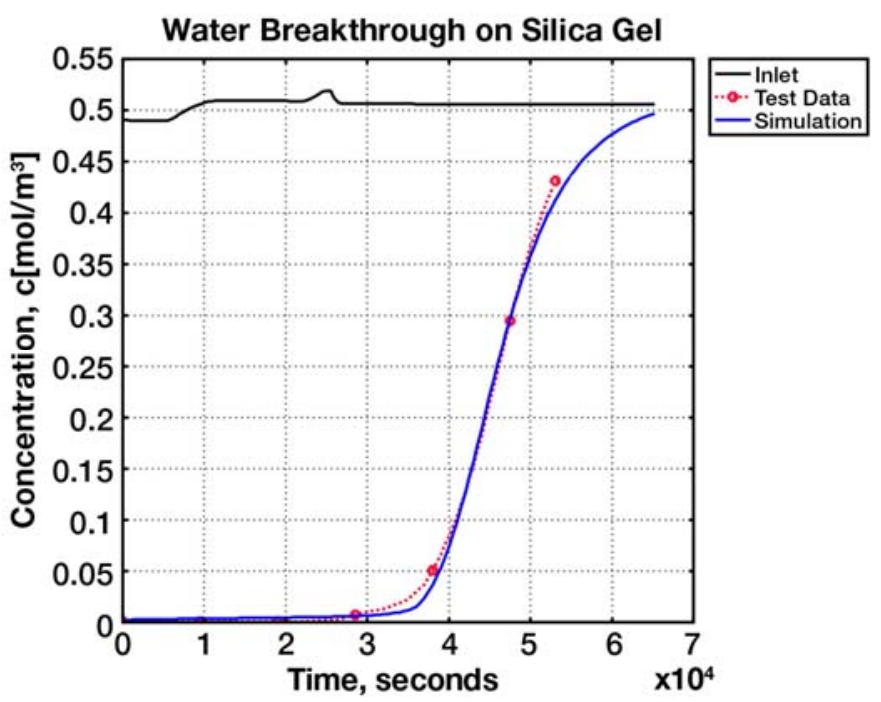

Figure 12: Water adsorption by silica gel. The actual outlet data are the dotted lines and simulation results are the solid red line.
These simulations indicated very good predictions from the adsorption model, the next step was to characterize the thermal characteristics of a sorbent canister and to determine the heat transfer coefficient between the fluid and the sorbent and the fluid and the wall. To do this, the apparatus shown in Figures $13 \mathrm{~A}$ and $13 \mathrm{~B}$ was used. $^{2}$

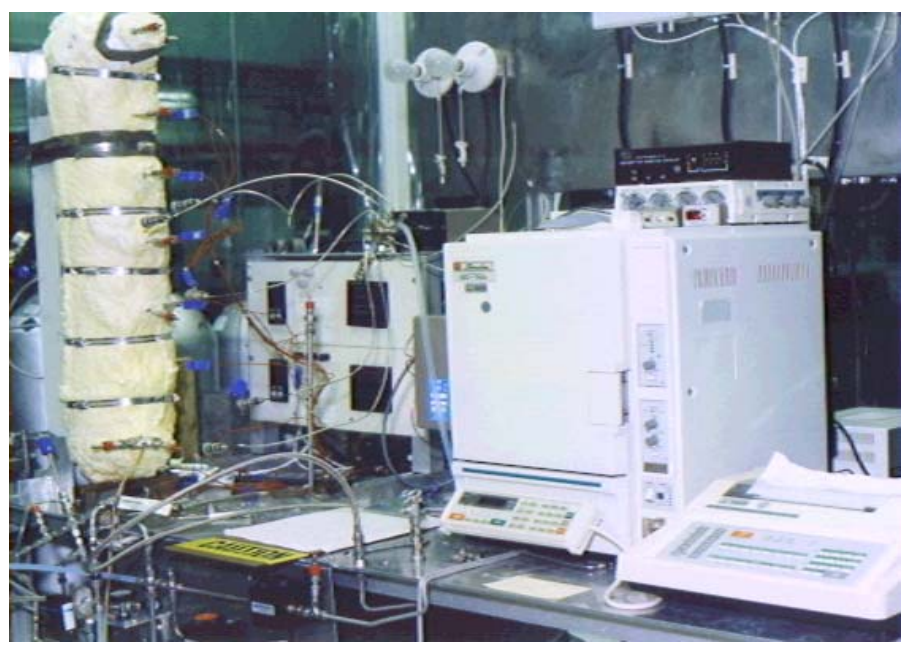

Figure 13A: The test apparatus used for determination of the heat transfer coefficient between the fluid and the sorbent and the fluid and the wall.
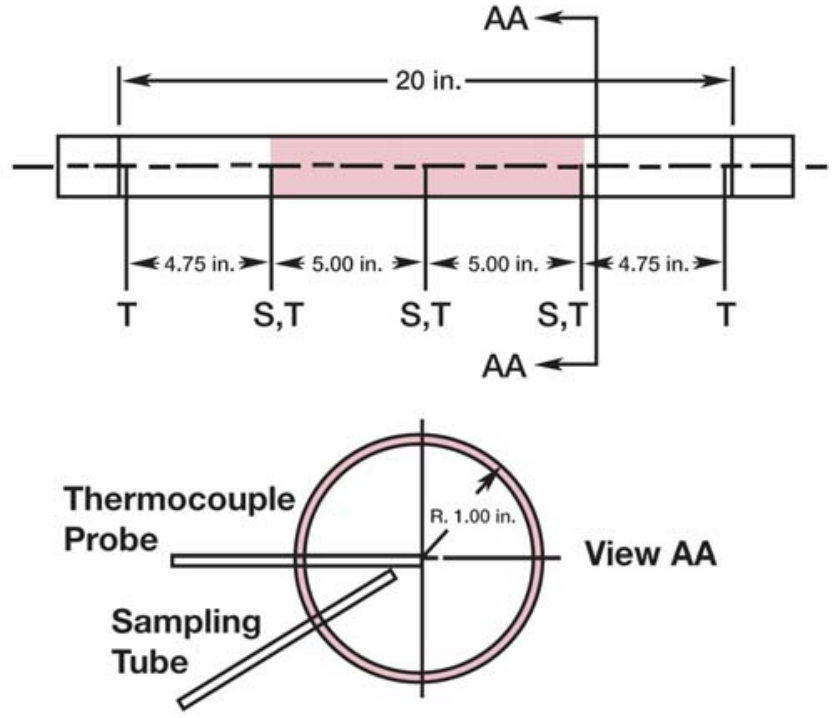

Figure 13B - A schematic representation of the test chamber, showing the locations of the sensing probes in the column of sorbent.

Looking at the heat balance for the gas phase, this testing started with a clean bed of sorbent material and introduced $450 \mathrm{~K}$ nitrogen, resulting in the large temperature swing shown in Figure 14. Modeling results following adjustment of the heat-transfer coefficients (also shown in Figure 14) provided a good match between the test results and the COMSOL Multiphysics 
simulation. This provided usable characterization of the system.

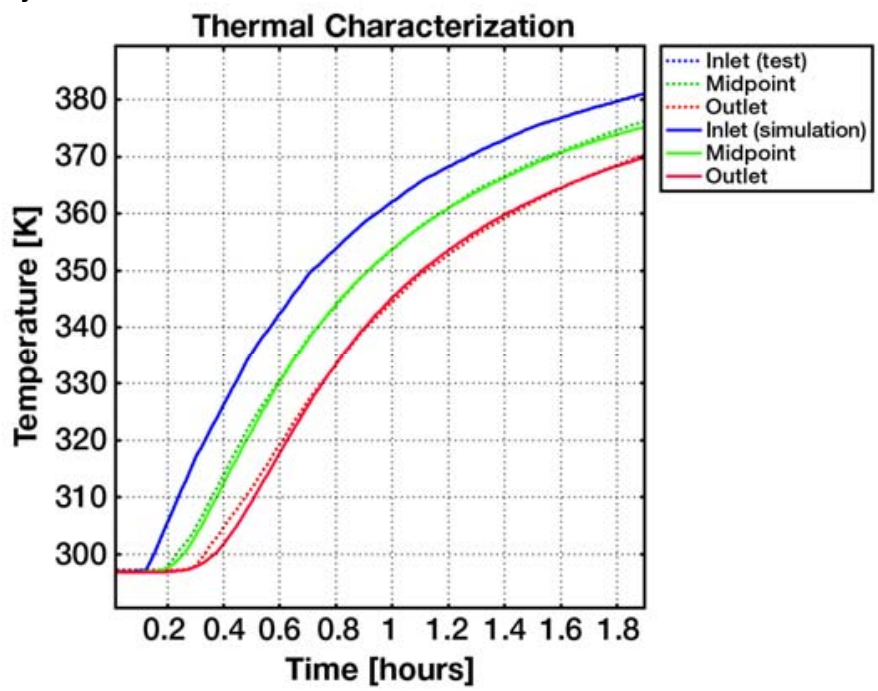

Figure 14: A comparison of test results and the COMSOL Multiphysics simulation characterized the heat transfer.

After some additional testing and simulations, it was shown that eliminating the heat of adsorption delays the initial breakthrough by about an hour, not an insignificant amount of time (see Figure 15). This confirms that by adjusting the actual adsorption cycle to take advantage of this delay, an increase in the adsorption performance and, hence, the working capacity can be realized.

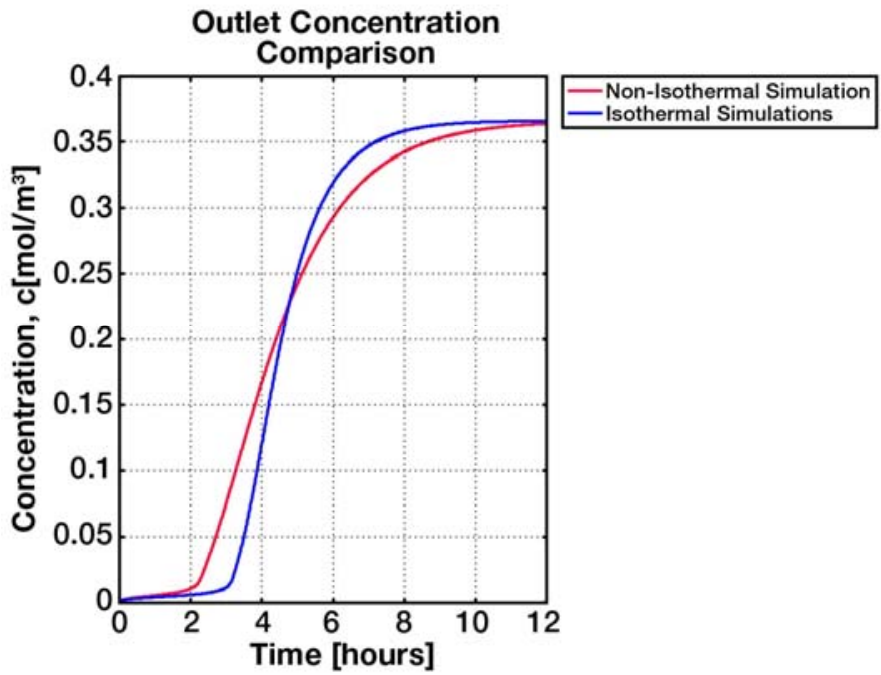

Figure 15: Simulation of Theoretical Efficiency Improvement via Isothermal Operations.

This backs up the improved performance achieved by approaching isothermal operations of the thermally linked silica gel packed in aluminum foam chamber discussed in an earlier section.

\section{MODELING OF LATTICE STRUCTURES}

Optimizing the hardware and adsorption process requires understanding the effect of varying substrate geometry (metal strand size and spacing), process (flow, desorption method, and cycle time), and canister design (sorbent types and quantities). Computer simulations are being developed to capture the effects of changing these parameters on the fluid dynamics, transient mass transfer, and transient heat transfer during the adsorption process.

Figure 16A shows a model of an interior lattice of an ESS part formed by Election Beam Melting rapid prototype manufacturing, a potential substrate for sorbent coating [5]. Because modeling such a part requires enormous amounts of time and computing resources, a simpler and easier to simulate alternative interior design was substituted (Figure 16B). The design features metal rods with a sorbent coating on their outside, with the rods connected thermally by a wall.

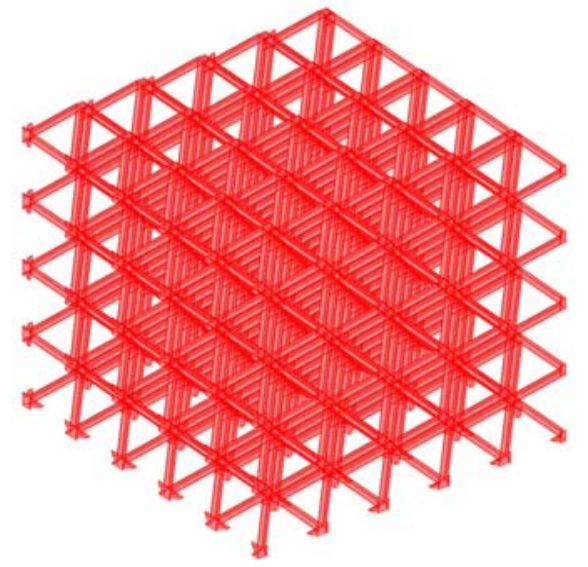

Figure 16A: The actual CAD file used to build an EBM lattice.

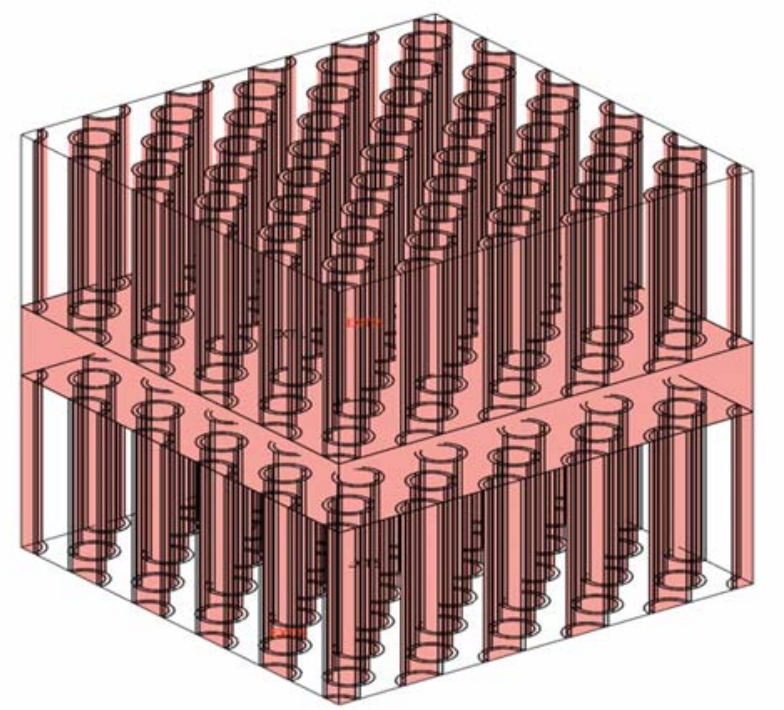

Figure 16B: An alternative and easier to model lattice showing metal rods coated in a sorbent and connected thermally by a wall in the middle.

Even the he alternate design was difficult to compute due to the large number of nodes created by the simulation. Consequently, a smaller subset of the design was looked at (Figure 17). The image in Figure 17 is a 2-dimensional COMSOL Multiphysics simulation of a Navier-Stokes incompressible steady-state analysis of the flow field around the rods. The results obtained show that, although the flow around the rods enters the 
adsorption chamber uniformly at $y=0$, it quickly forms an established, repeatable flow field.

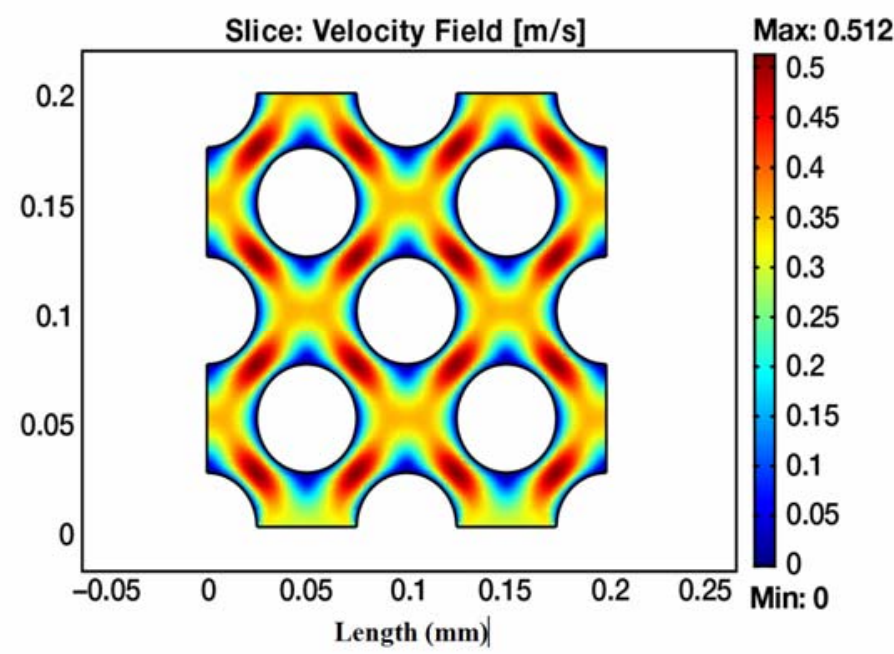

Figure 17: Image showing uniform fluid flow through a 2D structured sorbent lattice in the $\mathrm{Y}$ direction.

Next, the 3-dimensional flow was examined, specifically the boundary layer effects on the flow near the wall. Figure 18A shows the 3D simulation of flow through a bed of the structured sorbent lattice. The similarity of the flow pattern at varying distances from the wall at the bottom indicates the wall effect diminishes quickly away from the wall.

Figure 18B shows the flow field in the two exit planes along with the streamlines. Again, computer simulation shows that the flow field becomes very consistent a short distance away from the wall. This means that it is feasible to use a small portion of the full lattice to study the effects of changing rod size, spacing, and geometry on the fluid flow. The ultimate goal is to maximize the mass transfer from the fluid to the sorbent while minimizing the pressure differential through the bed.

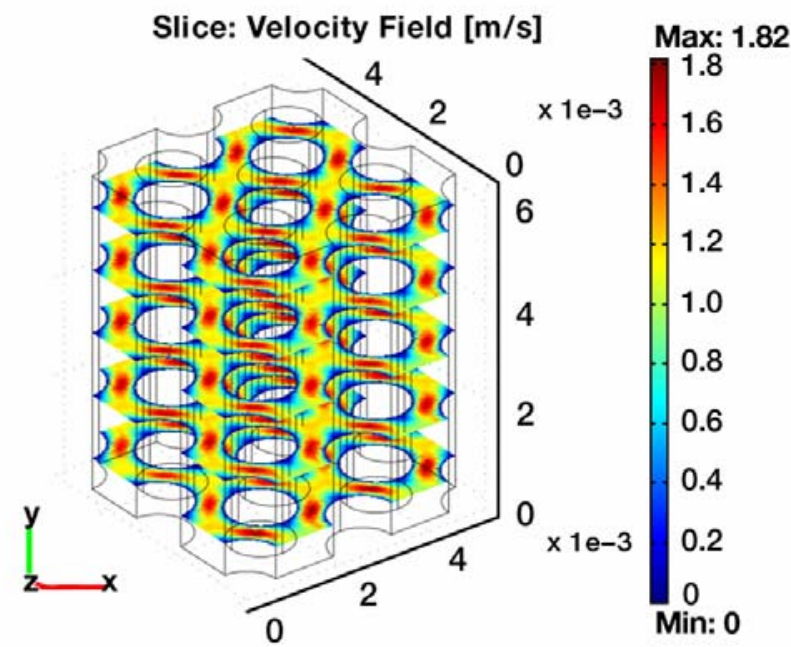

Figure 18A: 3D slice plot showing uniform fluid flow through a structured sorbent lattice. The length axes shown have an arbitrary unit.

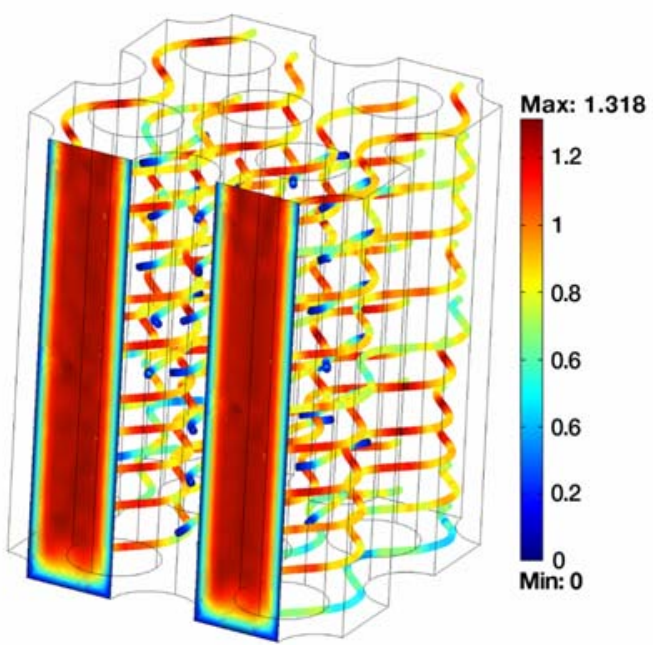

Figure 18B - A simulation of the 3D flow fluid. The boundary layers at the separating wall are evident in the bottom of the exit planes. Streamlines show highest velocity in constricted areas.

The developments in modeling thus far are a precursor to the desired modeling that combines thermal and fluid evaluations with sorption thermodynamic processes. Work continues to further develop modeling capabilities, primarily using COMSOL Multiphysics software.

\section{Conclusion}

Many ESS concepts are being considered in an effort to develop more efficient and robust solutions for air constituent control in future manned space applications. The advantages of fabricating such structures to allow for isothermal adsorption and direct contact to heating elements has been shown to significantly increase sorbent working capacity, and constructing sorbent structures to reduce or eliminate particle fines is paramount to increase robustness. To date, development of the presented technological concepts is at different stages, although all are considered still in their infancy. Several subscale examples have been produced with others to be completed in the near term. To fully understand the potential of these technologies, a combination approach of subscale testing and computer simulations will be carried out to evolve the most promising into full scale demonstration units, and eventually into technologies used in the next generation space habitats. 


\section{REFERENCES}

1. Elsherif, D., Knox, J. C., International Space Station Carbon Dioxide Removal Assembly (ISS CDRA) Concepts and Advancements, International Conference of Environmental Systems, 2005, 2005-01-2892

2. Perry, J., Roychoudhury, S., and Walsh, D., Microlith Based Sorber for Removal of Environmental Contaminants. International Conference of Environmental Systems, 2004, 2004-01-2442

3. United States Patent, Regenerable Adsorption System, Patent No. US 7,141,092 B1, Nov. 28, 2006, S. Roychoudbury, J. Perry, D. Walsh

4. Perry, J., Tomes, K., Roychoudhury, S., Tatara, J., Performance Characterization of a Prototype Ultra-Short Channel Monolith Catalytic Reactor for Air Quality Control Applications, International Conference of Environmental Systems, 2005, 2005-01-2868

5. Cooper, K. G., Good, J. E., Gilley, S.D., Layered Metals Fabrication Technology Development for Support of Lunar Exploration at NASA MSFC, Space Technology and Applications International Forum, 2007, 20070018826

6. Stuecker, S. N., Miller, J. E., Ferrizz, R. E., Mudd, J. E., Cesarano, J., Advanced Support Structures for Enhanced Catalytic Activity, Ind. Eng. Chem. Res. 2004, 43, 51-55

7. COMSOL Multi-physics modeling, www.comsol.com

8. Mohamadinejad, H., Knox, J.C., and Smith, J. E. Experimental and Numerical Investigation of Adsorption/Desorption in Packed Sorption Beds under Ideal and Nonideal Flows. Separation Science and Technology, 2000, 35(1), 1-22 